Bei diesem Obstipationstyp ist die Motilität des proximalen Kolon reduziert. Postprandiale hochamplitudige Kontraktionen fehlen oder sind in ihrer Ausprägung reduziert. Ursächlich spielen morphologisch nachweisbare Defekte des Plexus myentericus eine Rolle. „Je geringer die Stuhlfrequenz ist, desto schlechter sprechen diese Patienten auf Ballaststoffe und osmotisch wirksame Laxanzien an“, so Teich.

\section{Entleerungsstörungen sind am häufigsten}

Sehr viel häufiger ist die Obstipation aufgrund von Entleerungsstörungen. Hier beginnt der pathogenetische Weg meist mit der bewussten oder unbewussten Unterdrückung des Stuhlgangs. Dadurch kommt es zu einer Verdichtung und Verhärtung der Stuhlsäule im distalen Sigma und Rektum. Die nachfolgende Defäkation ist schmerzhaft und kann zu einer Analfissur oder einem Hämorrhoidalleiden führen. „Dies wiederum führt in der Folge zu einem gesteigerten Vermeidungsverhalten im Sinne eines Circulus vitiosus“, so Teich.

Sehr viel seltener sind Entleerungsstörungen durch strukturelle Veränderungen wie z. B. eine Rektozele oder eine exzessive Becken-Boden-Senkung, die eine operative Therapie erfordert.

\section{Meist normaler Kolontransit}

Bei den meisten Patienten mit chronischer Obstipation ist der Kolontransit nicht verlangsamt. Vielmehr passiert der Stuhl den Darm in normaler Geschwindigkeit und die Stuhlfrequenz unterscheidet sich auch nicht von Gesunden. Doch die Betroffenen berichten über Entleerungsschwierigkeiten, Blähungen und Bauchschmerzen. „Bei diesen Patienten dürfte eine erhöhte Rektumcompliance und eine erniedrigte Rektumsensitivität vorliegen“, so Teich. Deshalb sprechen solche Patienten häufig auf Ballaststoffe und/oder osmotisch wirksame Laxanzien gut an.

\section{Aufklärung über „das Normale“}

Bei mehr als der Hälfte der Patienten mit chronischer Obstipation finden sich

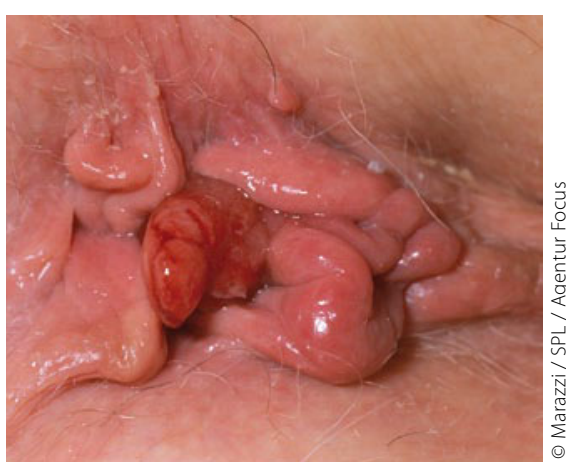

Mehr als lästige Folge chronischer Verstopfung: Hämorrhoiden. keine strukturellen Veränderungen. „Dies legt einen Einfluss mentaler und kognitiver Faktoren nahe“, so Teich. Doch die Datenlage zu dieser sich im Alltag immer wieder bestätigenden Annahme sei allerdings außerordentlich schlecht.

Nach offiziellen Kriterien gilt erst eine Stuhlfrequenz von weniger als drei Stühlen pro Woche als obstipiert. Obwohl fast $20 \%$ der Bevölkerung sich selbst als verstopft bezeichnen, gibt nur jeder Vierte davon weniger als drei Stuhlentleerungen pro Woche an. Die große Mehrheit betroffener Patienten empfindet bereits die Notwendigkeit zum Pressen und das Völlegefühl als führendes Problem im Sinne einer Obstipation. Deshalb ist, so Teich, die Aufklärung über „das Normale“ das vorrangige Therapieprinzip. Eine Kombination aus Aufklärung über kognitive und behaviorale Aspekte sowie einer möglichst nebenwirkungsfreien Ernährungs- und pharmakologischen Therapie sei die Basis eines erfolgreichen Patienten-Managements.

DR. med. Peter Stiefelhagen .

- Quelle: Symposium der Falk - Foundation e.V. im Rahmen des 117. Kongresses der deutschen Gesellschaft für Innere Medizin, 29.4.2011 in Wiesbaden.

\title{
Die Operation ist die Ultima Ratio
}

Eine chronische Obstipation kann Ausdruck verschiedener neurologischer, metabolischer oder endokrinologischer Erkrankungen sein. Auch an Nebenwirkungen von Medikamenten muss gedacht werden. Das Spektrum der dabei in Frage kommenden Substanzen reicht von Antihypertensiva wie Kalziumantagonisten über Parkinsonmedikamente bis hin zu Psychopharmaka. Bei den endokrinologischen Ursachen steht die Hypothyreose und die diabetische Neuropathie im Vordergrund.

Die gefürchtetste Ursache ist jedoch ein stenosierendes Dickdarmkarzinom, wes- halb immer eine totale Koloskopie angestrebt werden sollte.

Ist eine organische Ursache ausgeschlossen, spricht man von einer funktionellen Obstipation. „Leichtere Formen einer solchen funktionellen Obstipation können meist problemlos mit lebensdiätetischen Maßnahmen und/oder Laxanzien behandelt werden", so Priv.-Doz. Dr. Anton J. Kroesen, Köln. Nur bei einem kleinen Teil der Patienten mit einer "Slow-TransitObstipation" sei eine dezidierte Diagnostik und eventuell - nach langzeitiger frustraner medikamentöser Therapie - eine Operation indiziert. Die Therapie der
Wahl ist dann eine subtotale Kolektomie mit ileorektaler Anastomose. „Die Indikation für diesen Eingriff sollte äußerst zurückhaltend gestellt werden, da selbst nach ileorektaler Anastomose noch Fälle von persistierenden Entleerungsstörungen berichtet wurden", so Kroesen.

Unverzichtbar ist die chirurgische Intervention bei obstruktiven Defäkationsstörungen. Dazu gehört die Obstipation durch eine Behinderung der Rektumentleerung im Rahmen einer ausgeprägten Intussuszeption des Rektums oder einer anterioren Rektozele.

$\mathrm{STI}=$ 